\title{
Mathematical modeling and algorithm for calculation of thermocatalytic process of producing nanomaterial
}

\author{
Bakhtiyar Ismailov', Zhanat Umarova ${ }^{2}$, Khairulla Ismailov ${ }^{3}$, Aibarsha Dosmakanbetova ${ }^{4}$, \\ Saule Meldebekova ${ }^{5}$ \\ ${ }^{1,2,3}$ Department of Information Systems and Modeling, M. Auezov South Kazakhstan University, Kazakhstan \\ ${ }^{4}$ Department Technological machines and equipment, M. Auezov South Kazakhstan University, Kazakhstan \\ ${ }^{5}$ Department of Computing technology and software, M. Auezov South Kazakhstan University, Kazakhstan
}

\section{Article Info}

Article history:

Received Apr 18, 2021

Revised Jul 8, 2021

Accepted Aug 5, 2021

\section{Keywords:}

Laplace transform

Mathematical modeling

Nanomaterials

Numerical algorithm

Periodic and continuous modes

Thermocatalytic pyrolysis

\begin{abstract}
At present, when constructing a mathematical description of the pyrolysis reactor, partial differential equations for the components of the gas phase and the catalyst phase are used. In the well-known works on modeling pyrolysis, the obtained models are applicable only for a narrow range of changes in the process parameters, the geometric dimensions are considered constant. The article poses the task of creating a complex mathematical model with additional terms, taking into account nonlinear effects, where the geometric dimensions of the apparatus and operating characteristics vary over a wide range. An analytical method has been developed for the implementation of a mathematical model of catalytic pyrolysis of methane for the production of nanomaterials in a continuous mode. The differential equation for gaseous components with initial and boundary conditions of the third type is reduced to a dimensionless form with a small value of the peclet criterion with a form factor. It is shown that the laplace transform method is mainly suitable for this case, which is applicable both for differential equations for solid-phase components and calculation in a periodic mode. The adequacy of the model results with the known experimental data is checked.
\end{abstract}

This is an open access article under the CC BY-SA license.

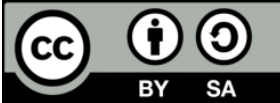

\section{Corresponding Author:}

Zhanat Umarova

Department of Information Systems and Modeling

M. Auezov South Kazakhstan University

Tauke khan avenue, 5, Shymkent, Kazakhstan

Email: zhanat.umarova@auezov.edu.kz

\section{INTRODUCTION}

Mathematical modeling of chemical technological processes is one of the science-intensive areas of knowledge. A large number of mathematical models known in the scientific literature are associated with the conditions for performing the process with fixed parameters-the dimensions and geometry of the apparatus, the values of temperature and pressure, the composition of the feedstock, and the type of catalyst. Researchers prove the solvability of models and the convergence of the applied numerical methods in different ways, so we can state the spread of methods for designing and implementing mathematical models. This circumstance creates difficulties in the application and comparison of the results of the implementation of models obtained by various researchers. In the field of chemical engineering, new challenges are emerging that require quick solutions. The creation of hydrocarbon-based nanomaterials (hereinafter NM) is of great interest. 
In this article, to continue the ideas of works [1]-[5], mathematical modeling of low-temperature synthesis of carbon nanostructures is carried out. The results of a comprehensive study of the structure and morphology of metal nanopowders synthesized by the method of the electric explosion of conductors, which were used as catalysts, are presented. In the course of the experiments, the technological parameters optimal for the low-temperature growth of carbon nanostructures were determined. In particular, the experimental regimes of low-temperature chemical vapor deposition of carbon nanotubes using iron and nickel nanopowders as a catalyst were found for the first time. Modern scientific developments in the field of synthesis of NM mathematical modeling using the catalytic pyrolysis method are described by two approaches:

- Modeling the formation of structural blocks and the process of NM growth, which provides for tracking the structure of NM (morphology) at the micro-level;

- Modeling the process of forming structural blocks at the macro level, providing an assessment of the performance of the apparatus.

These approaches are well known, and they are the main methods of a systematic approach to modeling the main processes of chemical production. The works [6]-[7] describe a new technology for producing carbon nanotubes and pure hydrogen by catalytic pyrolysis of hydrocarbon raw materials, the introduction of which opens up wide opportunities due to its cost-effectiveness. In our opinion, one should distinguish between "fast" and "slow" (diffusion type) technological processes. For type 1, rather high flow rates are characteristic, as a result of which vortex and turbulent flows are realized. Modeling of such problems is given, for example, in works [8]-[11]. To simulate technological processes, the methodology of works [12]-[14] can be applied. Difference schemes for parabolic equations with non-smooth boundary conditions and right-hand sides are modified in them. The problems of the correctness of the identification problem for the right-hand side of a parabolic equation were solved in [15]-[20]. Recently, new works have appeared on the modernization of Smoluchowski's theory [21]-[22], as applied to the problems of chemical technology for the synthesis of particles. In these works, the problems of flows modeling in technological devices and scaling when calculating the speed of particles in the binary aggregation are considered. Differential equations of various forms are widely used to describe the pyrolysis process and they include, among other things, expressions that take into account the influence of the concentrations of intermediate radicals, according to the stages of transformation of materials to finished NM. Currently, there are models of catalytic pyrolysis of natural gas together with the formation of NM in a continuous and variable tubular reactor. However, these models are not rigorously checked for their adequacy, since they characterize the process under study only for certain conditions and initial control data. The scientific novelty of this article is the method of obtaining an analytical representation for the carbon concentration with criterion parameters, which allows comparing the obtained modeling results with the data of other authors, with the justification of the existence and uniqueness of the solution of the formulated initial-boundary value problem for the differential equation.

\section{THE PROPOSED RESEARCH METHOD}

2.1. Development of a mathematical model of thermocatalytic pyrolysis form [7]:

Let's write the mathematical model of the thermocatalytic pyrolysis process in the following

$$
\begin{aligned}
& \frac{\partial C_{i}}{\partial t}+v_{1} \frac{\partial C_{i}}{\partial x}=D_{i} \frac{\partial^{2} C_{i}}{\partial x^{2}}+D_{i} \frac{\partial^{2} C_{i}}{\partial r^{2}}+\frac{D_{i}}{r} \frac{\partial C_{i}}{\partial r}+J_{i} \\
& C_{i}(t=0, x, r)=C_{i}^{0}(x, r) \\
& C_{i}(x=0, r)=C_{i}^{L}(r) \\
& \left.\frac{\partial C_{i}}{\partial x}\right|_{x=l}=0 \\
& \left.D_{i} \frac{\partial C_{i}}{\partial r}\right|_{r-0}=\sum_{j=1}^{M_{2}} v_{j}^{i} W_{j}, \text { if } x=l_{a p} / 2 \\
& \left.D_{i} \frac{\partial C_{i}}{\partial r}\right|_{r-0}=0, \text { if } x \neq l_{a p} / 2 \\
& \left.D_{i} \frac{\partial C_{i}}{\partial r}\right|_{r=D_{a p} / 2}=0
\end{aligned}
$$


Where $l_{a p}, D_{a p}$-length and diameter of the apparatus, m; $D_{i^{-}}$molecular diffusion coefficients for each $i$, $\mathrm{m}^{2} / \mathrm{sec} ; C_{i}^{L}$-initial concentration of the gas component at the reactor inlet, $\mathrm{mol} / \mathrm{m}^{3} ; \sum_{j=1}^{M_{2}} v_{j}^{i} W_{j}$ - the sum of the rates of formation and consumption of the i-component of the gas phase by reactions taking place on the catalyst surface, $\mathrm{mol} /\left(\mathrm{m}^{3 *} \mathrm{sec}\right) ; v_{j}^{i}$ - stoichiometric coefficient of the $i$-component of the gas phase in the $j$ surface reaction; $W_{j^{-}} j$-surface reaction rate, $\mathrm{mol} /\left(\mathrm{m}^{3}{ }^{*} \mathrm{sec}\right)$; $(1)$-a modified system of equations for the concentrations of gas components with an additional term that takes into account the effect of the concentrations of intermediate radicals; (2) initial conditions; (3), (4), (5), (6), (7)-boundary conditions for (1), taking into account the influx of reagents into the apparatus together with the initial mixture, the arrival-departure of gasphase components as a result of reactions on a catalyst located in the center of the apparatus. The equation for the change in the concentrations of the dispersed phase components:

$$
\frac{\partial C_{k, i}}{\partial t}=J_{k, i}
$$

Where $C_{k, i}$ - surface concentration of a solid phase component, reduced to unity catalyst mass, mol/ $\mathrm{kg} ; J_{k, i^{-}}$ the rate of formation or consumption of the $i$-component of the dispersed phase by reactions proceeding on the catalyst surface, $\mathrm{mol} /(\mathrm{kg} * \mathrm{sec})$.

The initial condition for (8) has the form:

$$
C_{k, i}(t=0)=C_{k, i}^{0}
$$

Where $C_{k, i}^{0}$ - the concentration of the $i$-component of the dispersed phase at the initial moment of time, mol $/ \mathrm{kg}$ (at the initial moment of time, the concentration of all components of the dispersed phase is zero, the catalyst activity is maximum). For the convenience of solving and comparing the results with the results of other authors, we turn to dimensionless variables: let $x^{*}$-the characteristic linear size of the apparatus; $c_{i}^{*}$-the average value of the concentration of the component; $t^{*}$ - the average time of the process; $r^{*}$ - the characteristic value of the radial direction. Dimensionless values are marked with a dash. Thus, we obtain the following system of equations for (1):

$$
\frac{\partial \bar{c}_{i}}{\partial \bar{t}}+R_{x} \frac{\partial \bar{c}_{i}}{\partial \bar{x}}=K_{x i} \frac{\partial^{2} \bar{c}_{i}}{\partial \bar{x}^{2}}+K_{r i}\left(\frac{\partial^{2} \bar{c}_{i}}{\partial r^{2}}+\frac{1}{r} \frac{\partial \bar{c}_{i}}{\partial r}\right)+\bar{\alpha}_{i-1} \bar{c}_{i-1}+\bar{\beta}_{i+1} \bar{c}_{i+1}+\bar{J}_{i}
$$

Where $R_{x}=\frac{t^{*} v_{1}}{x^{*}}$ - dimensionless number characterizing the length of the apparatus; $K_{x i}, K_{r i}-$ are the diffusion parameters of the components over $x, r ; \bar{\alpha}_{i-1}=\frac{\alpha_{i-1} t^{*}}{c_{i-1}^{*}}, \bar{\beta}_{i+1}=\frac{\beta_{i-1} t^{*}}{c_{i+1}^{*}}$ - dimensionless concentration factors; $\bar{J}_{i}=\frac{J_{i} t^{*}}{\bar{c}_{i}^{*}}$ - the relative rate of formation of the $i$-component in the reaction in the gas phase; within the meaning of the notation $-\bar{\alpha}_{0}=0, \beta_{N+1}=0$. Initial and boundary conditions (2)-(7), equations and conditions for the solid phase remain practically unchanged. The mathematical model-(2)-(9)-(10) can be realized using analytical or numerical methods. At the first stage, we will consider a simpler mode - continuous.

In this work, a mathematical model of the catalytic pyrolysis process will be implemented based on the laplace transform (small initial concentration of the gas component) and a numerical method (large values of the initial concentration). Theoretical and practical questions of the solvability and uniqueness of the solution of differential equations, which are most often used in the problem considered in this paper, are described in sufficient detail in [23]-[24]. The equation for the change in the concentration of the gas phase component in the one-dimensional formulation takes the form:

$$
\frac{\partial c}{\partial t}+v_{1} \frac{\partial c}{\partial x}=\mathrm{D}_{\mathrm{md}} \frac{\partial^{2} c}{\partial x^{2}}+J^{*}, 0<x<l, 0<t<T
$$

Initial condition:

$c(x, 0)=c^{0}(x)$

Border conditions:

$c(0, t)=c_{v}$-That is, the concentration at the inlet of the apparatus

$\left.\frac{\partial c}{\partial x}\right|_{x-l}=0$ - The concentration at the end of the apparatus does not change 
Here $l$ is the length of the apparatus, $\mathrm{m} ; v_{1}$-gas flow rate, $\mathrm{m} / \mathrm{sec} ; D_{m d}$-molecular diffusion coefficient, $\mathrm{m}^{2} / \mathrm{sec}$; $c$-gas concentration in the reactor, $\mathrm{mol} / \mathrm{m}^{3} ; J^{*}$ - the rate of formation or consumption of a component in the gas phase, $\mathrm{mol} /\left(\mathrm{m}^{3} * \mathrm{sec}\right)$.

For the convenience of calculations, let us pass to dimensionless variables, choosing characteristic values for each of them. After transformations, we get (the dashes over the variables are omitted):

$$
\frac{\partial c}{\partial t}+\frac{\partial c}{\partial x}=P e^{-1} * F * \frac{\partial^{2} c}{\partial x^{2}}+J
$$

Where $P e=v_{1} l / D_{m d}$ - Peclet criterion; $F=L / D$-geometric factor of the apparatus shape; $\mathrm{b}=\mathrm{F} / \mathrm{Pe}=$ const, $J$ is the dimensionless rate of formation of the gas phase of the components for the reaction taking place on the catalyst surface (constant value in the isothermal formulation of the problem).

Initial condition:

$$
c(x, 0)=c^{0}(x)
$$

In a particular case, in (16) could be taken:

$$
c^{0}(x)=c_{0}=\text { const }
$$

Border conditions:

$c(0, t)=c_{i n}$-Inlet concentration

$\left.\frac{\partial c}{\partial x}\right|_{x=1}=0$-The concentration at the end of the apparatus does not change

If $c^{0}(x) \cong 0$, then the problem is formulated as follows:

$$
\begin{aligned}
& b \frac{\partial^{2} c}{\partial x^{2}}-\frac{\partial c}{\partial x}-\frac{\partial c}{\partial t}=-J \\
& c^{0}(x, 0)=c_{0}(x) \\
& c(0, t)=c_{0} \\
& \left.\frac{\partial c}{\partial x}\right|_{x=1}=0 \\
& b=F / P e=\text { const }
\end{aligned}
$$

Note: Problem (20)-(23) can be formulated and solved according to the methodology presented in [12]-[20]. Namely, in a more general setting, we represent this problem in the form:

$$
\begin{aligned}
& \frac{\partial c}{\partial t}-\alpha \frac{\partial^{2} c}{\partial x^{2}}+\beta \frac{\partial c}{\partial x}=f(x, t), 0<t<T, 0<x<1 \\
& c(x, 0)=\phi(x), 0 \leq x \leq 1 \\
& c(0, t)=\omega(t), 0 \leq t \leq T \\
& \frac{\partial c(1, t)}{\partial x}=0
\end{aligned}
$$

We introduce a new function $v(x, t)$, so that homogeneous boundary conditions are satisfied: $c(x, t)=$ $v(x, t)+\mu(x, t)$, where $\mu(x, t)=x^{2}-2 x$.

Then problem (25)-(28) takes the following form:

$$
\frac{\partial v}{\partial t}-\alpha \frac{\partial^{2} v}{\partial x^{2}}+\beta \frac{\partial v}{\partial x}=f(x, t), 0<t<T, 0<x<1
$$




$$
\begin{aligned}
& v(x, 0)=\varphi_{1}(x), 0 \leq x \leq 1 \\
& v(0, t)=0,0 \leq t \leq T \\
& \frac{\partial v(1, t)}{\partial x}=0,0 \leq t \leq T
\end{aligned}
$$

Initial condition:

$y(x, 0)=c(x, 0)-x^{2}+2 x$

The functions $v(x, t)$ and $c(x, t)$ are related by the relation:

$v(x, t)=c(x, t)-x^{2}+2 x$

Hence, for (1) we get:

$f(x, t)=\frac{\partial c}{\partial t}-\alpha \frac{\partial^{2} c}{\partial x^{2}}+\beta \frac{\partial c}{\partial x}=J+2 \alpha+2 \beta(1-x)$

The task takes the following form:

$$
\begin{aligned}
& \frac{\partial v}{\partial t}-\alpha \frac{\partial^{2} v}{\partial x^{2}}+\beta \frac{\partial v}{\partial x}=J+2 \alpha+2 \beta(1-x), 0<t<T, 0<x<1 \\
& v(x, 0)=c_{0}-x^{2}+2 x, 0 \leq x \leq 1 \\
& v(0, t)=0,0 \leq t \leq T \\
& \frac{\partial v(1, t)}{\partial x}=0,0 \leq t \leq T
\end{aligned}
$$

Thus, the original problem is reduced to the problem (36)-(39), for which the conditions for the existence and uniqueness of the solution were proved in [12]-[14]. Therefore, we can assume that the equivalent problem (20)-(23) has a unique solution.

\subsection{Solution of the problem by the Laplace transform method}

We solve the initial boundary value problem by applying the Laplace transform:

$$
L(c(x, t), x, t)=v=\int_{0}^{\infty} e^{-p t} c(x, t) d t
$$

Where $p$ is a number with a large enough positive real part for the integral to converge. Applying the Laplace transform to (20)-(23), we obtain the following boundary value problem for an ordinary differential equation:

$$
\begin{aligned}
& b \frac{d^{2} v}{d x^{2}}-\frac{d v}{d x}-p v=-\frac{J}{p} \\
& v(0)=0 \\
& \left.\frac{\partial v}{\partial x}\right|_{x=1}=0
\end{aligned}
$$

Let's write the characteristic equation for the homogeneous in (41) as follows:

$b k^{2}-k-p=0$

Where $k_{1,2}=\frac{1 \pm \sqrt{1+4 b p}}{2 b}$

Let:

$\alpha=\frac{1+\sqrt{1+4 b p}}{2 b}, \beta=\frac{1-\sqrt{1+4 b p}}{2 b}$ 
Then:

$v(x)=c_{1} e^{\alpha x}+c_{2} e^{\beta x}$

The particular solution of the inhomogeneous equation could be found from the (41).

Let's $\bar{v}=z$, then $p z=\frac{-J}{P}, z=\frac{J}{p^{2}}$

$v(x, p)=c_{1} e^{\alpha x}+c_{2} e^{\beta x}+\frac{J}{p^{2}}$

$c_{1}+c_{2}=-\frac{J}{p^{2}}, c_{1} \alpha+c_{2} \beta=0$

The solutions of system (48) are the following numbers:

$c_{1}=-\frac{\beta J}{p^{2}(\beta-\alpha)}, c_{2}=\frac{\alpha J}{p^{2}(\beta-\alpha)}$

Then the required function is equal to:

$v(x, p)=\frac{J}{p^{2}(\beta-\alpha)}\left(\alpha e^{\beta x}-\beta e^{\alpha x}\right)+\frac{J}{p^{2}}$

For further calculations, we take into account the following ratio:

$\beta-\alpha=-\frac{\sqrt{1+4 b p}}{b}$

Then it can be written as:

$v(x, p)=\frac{J b}{p^{2} \sqrt{1+4 b p}}\left(\beta e^{\alpha x}-\alpha e^{\beta x}\right)+\frac{J}{p^{2}}$

Let's perform the inverse Laplace transform:

$c(x, t)=\frac{1}{2 \pi i} \int_{-i \infty}^{i \infty} v(x, p) e^{p t} d p$

To calculate the integral in (53), there is could be used the theory of residues:

$\frac{1}{2 \pi} \int_{-\infty}^{i \infty} v(x, p) e^{p t} d p=\sum_{i=1}^{N} r e z_{i} F(p)$

For function $v(x, t)$ :

$\operatorname{rez} f(z)=\frac{d}{d p}\left(\frac{1}{p^{2}} v(x, p) e^{p t}\right)=\frac{d}{d p}\left(J b \frac{\beta e^{\alpha x+p t}-\alpha e^{\beta x+p t}+J}{\sqrt{1+4 b p}}\right)$

Derivatives for $\alpha$ and $\beta$ are calculated by formulas (55):

$\frac{d \alpha}{d p}=\frac{1}{\sqrt{1+4 b p}}, \frac{d \beta}{d p}=-\frac{1}{\sqrt{1+4 b p}}$

At the pole point, we obtain the following solution:

$c(x, t)=J b\left(1-e^{x / b}-\frac{t-x}{b}\right)$

If the assumption about the smallness of the initial concentration $c^{0}(x) \cong 0$ is inappropriate, then technical difficulties arise for solving equation: 


$$
\frac{\partial c}{\partial t}+\frac{\partial c}{\partial x}=P e^{-1} * F * \frac{\partial^{2} c}{\partial x^{2}}+J
$$

By the Laplace transform method. Therefore, it is necessary to apply finite difference methods. The issues of numerical modeling of heat and mass transfer processes, convergence, and stability of finite-difference schemes (Fourier method) from a practical point of view are presented in [9]. Then we followed the recommendations of this work.

\subsubsection{Explicit scheme}

For convenience, we denote:

$$
F / P e=b
$$

Indexing of grid points: $i$-by $x, j$-by $t$. We approximate the first-order derivatives for $x$ and $t$ according to the "forward" scheme, and the second-order derivative for $x$ by the central difference. We obtain the following explicit scheme for the equation:

$$
\frac{c_{i, j+1}-c_{i, j}}{\tau}+\frac{c_{i+1, j}-c_{i, j}}{h}=b \frac{c_{i+1, j}-2 c_{i, j}+c_{i-1, j}}{h^{2}}+J_{i, j}
$$

When approximating the derivatives of the 1-order, the error is $O(\tau)+O(h)$, and for the derivative of the 2order $-O\left(h^{2}\right)$. After transformations we get:

$$
c_{i, j+1}=k_{1} c_{i-1, j}+k_{2} c_{i, j}+k_{3} c_{i+1, j}+\tau J
$$

Where:

$$
i=1, \ldots, n-1 ; j=0, \ldots, m-1 ; k_{1}=\frac{b \tau}{h}, k_{2}=1-\frac{2 b \tau}{h^{2}}+\frac{\tau}{h}, k_{3}=\frac{b \tau}{h^{2}}-\frac{\tau}{h}+J \tau, h=1 / n, \tau=1 / m
$$

Discrete analogues of conditions (21), (22), and (23) have the following form:

$$
c\left(x_{i}, 0\right)=c_{i, 0}, c\left(0, t_{i}\right)=c_{0, i} . c_{n, j}=c_{n-1, j}
$$

In the considered isothermal regime, $J=$ const. form:

The conditions for non-negativity $k_{1}, k_{2}, k_{3}$ and stability of scheme (61) taken together have the

$$
\tau / h^{2} \leq 1
$$

At large Peclet numbers $P e=v_{1} l / D_{m d}$, when convective transfer prevails over the molecular transfer, condition (63) takes the following form:

$$
\tau \leq c h^{2}
$$

Where the constant $\mathrm{c}$ decreases with increasing Peclet number. For example, at $P e \approx 1000: \mathrm{c} \approx 0,1$.

Such a smoothing procedure is often used and makes it possible to ensure a slow and smooth change in the calculated dynamic function. In our work, the Peclet number is small due to the predominance of the molecular component (i.e., on the order of 10); therefore, calculations using condition (65) were not performed. Taking into account the fact that the synthesis process is long, the requirement that condition (64) be satisfied concerning the time step leads to a large number of iterations, which results in the accumulation of round-off errors.

\subsubsection{Implicit scheme}

For large Peclet numbers, when convective matter transfer prevails, the use of an explicit scheme also leads to computational instability. For large peclet numbers, when convective matter transfer prevails, the use of an explicit scheme also leads to computational instability. Application to equation, the implicit scheme "backward" in time leads to the following problem: 


$$
\begin{aligned}
& \frac{c_{i, j}-c_{i, j-1}}{\tau}+\frac{c_{i+1, j}-c_{i, j}}{h}=b \frac{c_{i+1, j}-2 c_{i, j}+c_{i-1, j}}{h^{2}}+J_{i, j} \\
& c\left(x_{i}, 0\right)=c_{i, 0}, c\left(0, t_{j}\right)=c_{0, j} \cdot c_{n, j}=c_{n-1, j}
\end{aligned}
$$

The rate of formation or consumption of a component in the gas phase at all points of the reactor can be considered the same, i.e $J_{i j}=J$. Let us reduce the problem (66)-(67) to matrix form:

$-b \tau c_{i-1, j}+\left(h^{2}-\tau h+2 b \tau\right) c_{i, j}+(\tau h-b \tau) c_{i+1, j}=h^{2} c_{i, j-1}+\tau h^{2} J$

i.e., in matrix form:

$A_{i-1} c_{i-1}+A_{i} c_{i}+A_{i+1} c_{i+1}=B_{i}$

Where:

$$
A_{i-1}=-b \tau, A_{i}=h^{2}-\tau h+2 b \tau, A_{i+1}=\tau h-b \tau, B_{i}=h^{2} c_{i, j-1}+\tau h^{2} J
$$

To solve system (69) $A x=b$ by a tridiagonal matrix, we use the sweep method. For the applicability of the formulas of the sweep method, we check the conditions for the inequality of denominators to zero in the formulas for calculating the sweep coefficients and the diagonal dominance of the matrix A according to the formula:

$$
\left|h^{2}-\tau h+2 b \tau\right| \geq|-b \tau|+|\tau h-b \tau|
$$

For large Peclet numbers, i.e. for small $b$, we choose the step in $\mathrm{x}$ from the condition $2 \mathrm{~b}>\mathrm{h}>\mathrm{b}$, the step in time from the condition $\tau<$ h. Then the left-hand side of inequality (71) has the estimate:

$$
\left|h^{2}-\tau h+2 b \tau\right|>\tau h
$$

The right-hand side for $h>b$ is:

$$
|-b \tau|+|\tau h-b \tau|=b \tau+\tau(h-b)=\tau h
$$

Thus, when sufficiently weak conditions are satisfied for the steps of the mesh domain, the sweep method is convergent. We applied the proposed approach to the problem of determining the concentration of the components of a system with a $\mathrm{Ni} / \mathrm{MgO}$ catalyst along the length of the apparatus.

\subsection{Selection of catalysts and comparison of their parameters}

Various catalysts are used to accelerate the synthesis of nanostructures. Here two interrelated tasks arise-the selection and manufacture of the catalyst. In [25]-[27], monolithic catalysts based on $\mathrm{Co}, \mathrm{Ni}$, ordered mesoporous carbons, and $\mathrm{Ni} / \mathrm{MgO}$ catalysts on structured metal supports are used to produce hydrogen. Kirillov et al. [27] proposed to apply $\mathrm{Ni} / \mathrm{MgO}$ on a metal support, but this procedure is expensive and justifies itself for high-temperature synthesis in work [27]. Therefore, for use in our experimental studies, when the temperature does not exceed $7000 \mathrm{C}$, we have developed the following catalyst manufacturing technique: for the synthesis of a Ni-Mg catalyst, we impregnated alumina extrudates with solutions of active components-multiple nickels and magnesium nitrate. For this, aluminum hydroxide was mixed with distilled water. The resulting pasty mass was passed through a laboratory screw press-extruder. Then the extrudates were dried at room temperature, then dried and calcined at $600^{\circ} \mathrm{C}$ in atmospheric air. The resulting cylindrical granules have a diameter of 2.5-3 $\mathrm{mm}$ and a length of 5-6 $\mathrm{mm}$.

The catalyst samples synthesized in this way contain the active components $\mathrm{NiO}$ and $\mathrm{MgO}$ up to $10 \%$ each. We also studied the physicochemical indicators of the samples: specific and active surface; concentration of active centers; strength factor; total acidity. The data obtained are in satisfactory agreement with the data of works [23]-[24].

\section{RESULTS AND DISCUSSION}

Figure 1 shows the distribution of the concentration of methane concentration at the outlet from a batch reactor for small Peclet numbers, i.e. when molecular diffusion predominates. The calculation was made for methane, the physicochemical parameters of which have been studied in sufficient detail. As can be 
seen from Figure 2, at large values of the Peclet criterion (i.e., the ratio of dynamic and diffusion parameters), the methane concentration changes slowly. This is due to the predominance of the linear velocity of the gas over the rate of the diffusion process.

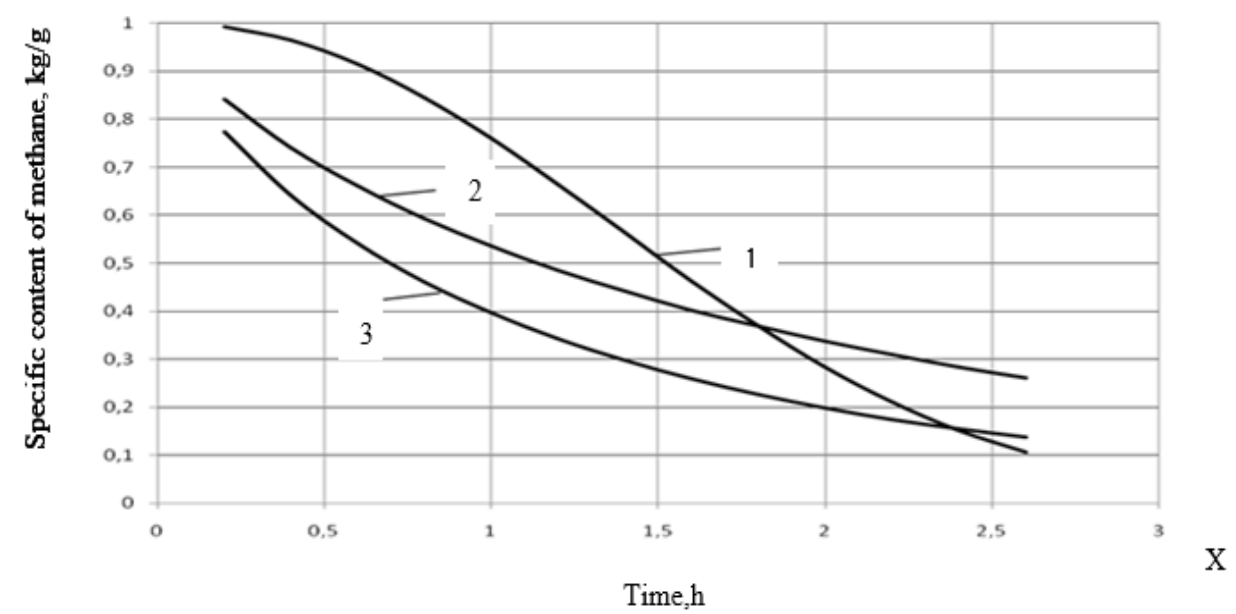

Figure 1. Distribution of methane concentration at different values of the Peclet criterion over Ni/MgO catalyst in time at temperature $600^{\circ} \mathrm{C}: 1-\mathrm{Pe}=10,2-\mathrm{Pe}=20,3-\mathrm{Pe}=30$

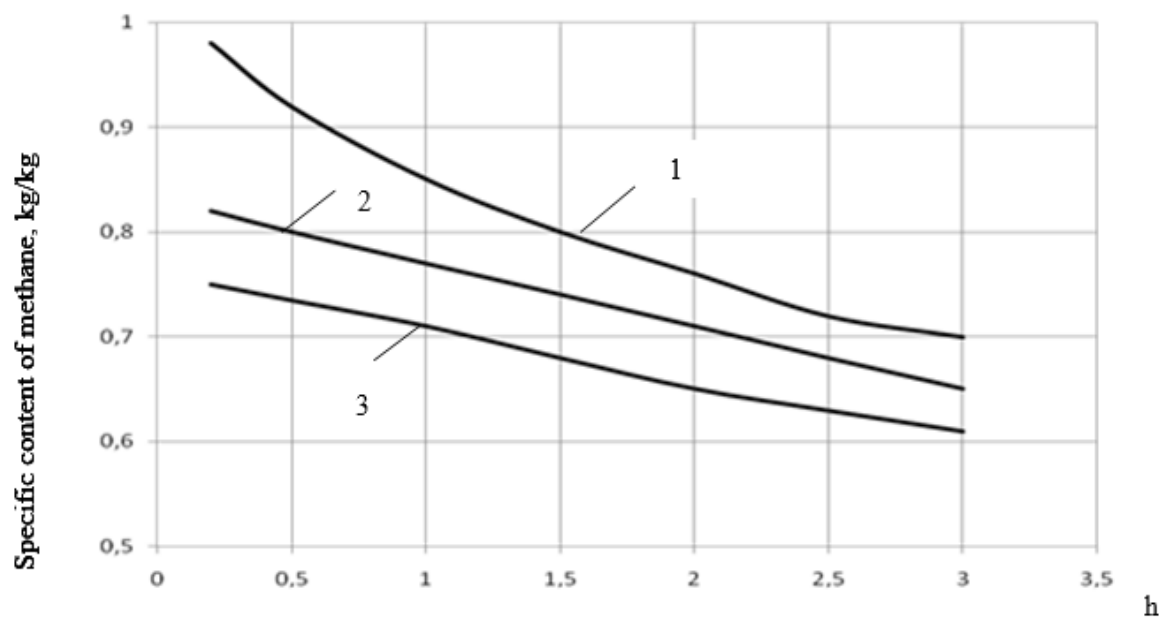

Figure 2. Distribution of methane concentration at different values of the Peclet criterion over $\mathrm{Ni} / \mathrm{MgO}$ catalyst in time at temperature $600^{\circ} \mathrm{C}: 1-\mathrm{Pe}=200,2-\mathrm{Pe}=300,3-\mathrm{Pe}=400$

As can be seen from Figure 2, at large values of the Peclet criterion (i.e., the ratio of dynamic and diffusion parameters), the methane concentration changes slowly. This is due to the predominance of the linear velocity of the gas over the rate of the diffusion process. We carried out a systematic numerical experiment using our sweep program, which implements the above sweep algorithm, to calculate the distribution of the specific carbon content on the catalyst. At the same time, to ensure the possibility of comparing the obtained numerical results with known experimental data [6]-[7], the actual type of catalyst, temperature values, and a set of values of the physicochemical parameters of the thermocatalytic pyrolysis process were selected. Figures 3-4 show the distribution of the specific carbon content calculated using the above algorithm. The relative error in calculating the specific carbon content using a Ni/MgO catalyst is $5 \%$. Changes in the concentration of methane and an increase in the concentration of carbon nanoparticles (Figures 1-4) are in agreement with the conclusions of [6]-[7]. 


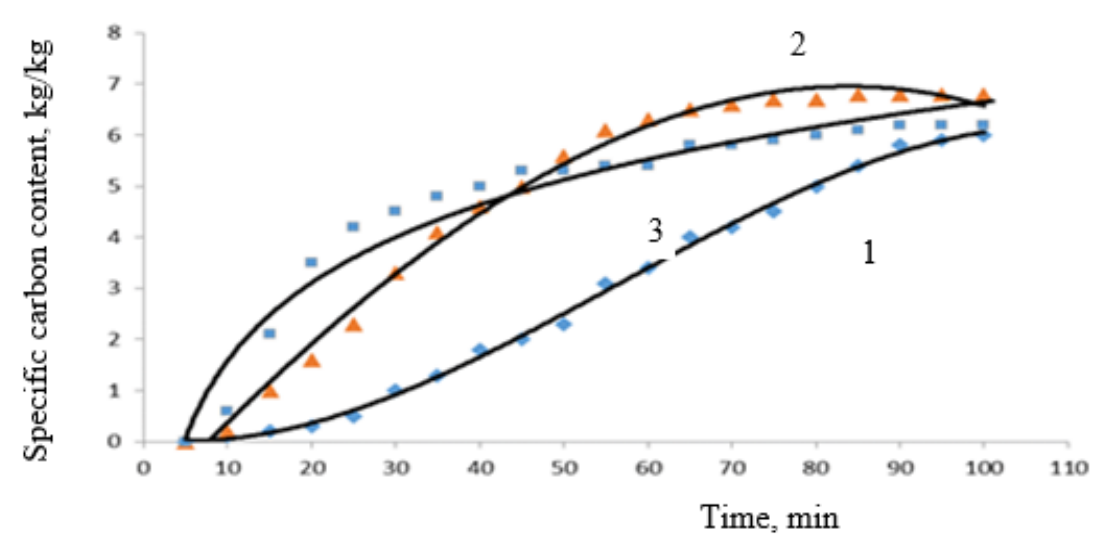

Figure 3. Calculated distribution of the specific carbon content on the $\mathrm{Ni} / \mathrm{La}_{2} \mathrm{O}_{3}$ catalyst over time at a temperature of $600^{\circ} \mathrm{C}$, (curve 1), $650^{\circ} \mathrm{C}$, (curve 2), $700^{\circ} \mathrm{C}$, (curve 3), points - experimental data [6]-[7]

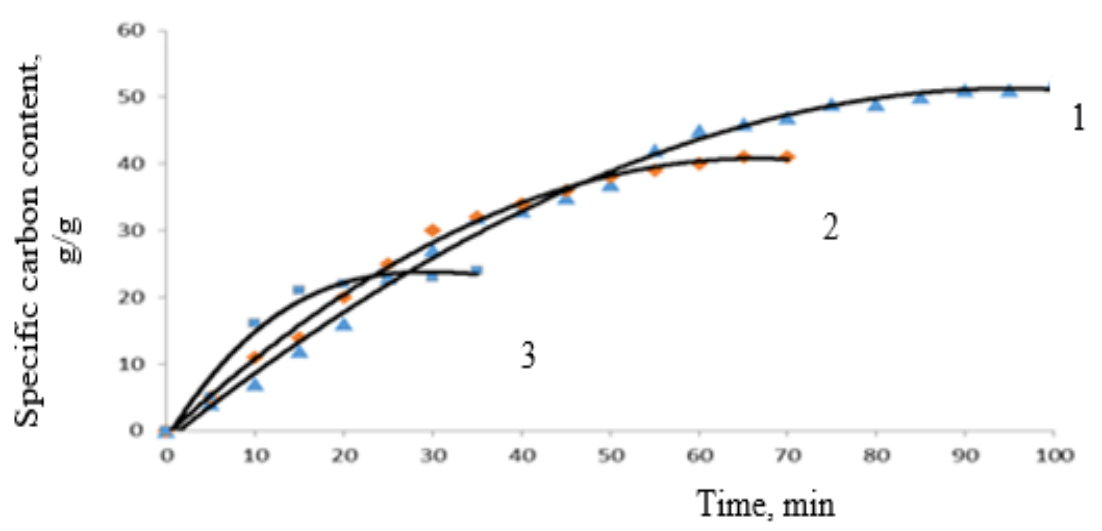

Figure 4. The calculated distribution of the specific carbon content on the $\mathrm{Ni} / \mathrm{MgO}$ catalyst over time at a temperature of $560^{\circ} \mathrm{C}$, (curve 1), $580^{\circ} \mathrm{C}$, (curve 2), $680^{\circ} \mathrm{C}$, (curve 3), points are experimental data [6]-[7]

\section{CONCLUSION}

A one-dimensional problem of mathematical modeling of the thermocatalytic process of obtaining nanomaterials is formulated, methods of implementing a mathematical model by the numerical method and the Laplace transform are developed. Such a statement of the problem was carried out for the first time and can be developed for use in research and production purposes in the production of nanomaterials. The distributions of methane concentration were obtained for different values of the Peclet diffusion number. The conditions of stability of numerical algorithms for the equation of concentration of gas components are established. The presented algorithm for the numerical simulation of the process of thermocatalytic synthesis of carbon nanomaterials, in our opinion, can be generalized for the case of the mutual influence of intermediate radicals on the product yield. To do this, it is necessary to develop a kinetic scheme of reactions, solve the differential equations for the transformations of $\mathrm{CH} 4-\mathrm{CH} 3-\mathrm{CH} 2-\mathrm{CH}-\mathrm{CH}$ with initial and boundary conditions, include additional terms in the system, $(i=1, \ldots, n-1$,$) , taking into account the influence$ concentrations of intermediate radicals on the rate of catalysis. Considering the fact that the process of thermal catalysis of carbon pyrolysis is a slow and, on average, is realized in several tens of hours, the method of mathematical modeling followed by implementation by numerical algorithms can significantly reduce time and material costs, as well as improve the quality of the resulting nanomaterials. The analytical formula for the concentration distribution in the form (57) was obtained for the first time and it is more accurate than the results of numerical solutions using existing techniques. When calculating the concentration, the value of the criterion ratio $b=F / P e$ is involved as a parameter, which makes it possible to carry out a systematic numerical experiment to formulate and solve the problems of optimizing the operating characteristics of the catalytic pyrolysis process and the geometric dimensions of the technological apparatus. 


\section{REFERENCES}

[1] E. G. Rakov, I. G. Ivanov, S. N. Blinov, N. V. Kazakov, V. V. Skudin, N. G. Digurov, and A. K. Bogdanovich, "Kinetics of Carbon Multi-wall Nanotube Synthesis by Catalytic Pyrolysis of Methane," Fullerenes, Nanotubes and Carbon Nanostructures, vol. 12, no. 1, pp. 29-32, Aug. 2006, doi: 10.1081/FST-120027129.

[2] G. Partizan, B. Z. Mansurov, B. S. Medyanova, A. B. Koshanova, M. E. Mansurova, B. A. Aliyev, and X. Jiang, "Low-temperature synthesis of carbon nanotubes on iron nanopowders," Materials Research Express, vol.3, no.11, pp. 123-130, Nov. 2016, doi: 10.1088/2053-1591/3/11/115010.

[3] Z. A. Mansurov, "Soot and Nanomaterials Synthesis in the Flame," Journal of Materials Science and Chemical Engineering, vol. 2, no.1, pp. 1-6, Jan. 2014, doi: 10.4236/msce.2014.21001.

[4] Z. A. Mansurov, "Obtaining of Nanomaterials in Combustion Processes," Advanced Materials Research, vol. 486, pp.134-139, Mar. 2012, doi: 10.4028/www.scientific.net/AMR.486.134.

[5] I. S. Nenaglyadkin, S. Yu, N. Tsavera, and E. G. Rakov, "Mathematical simulation of catalitical pyrolysis for carbon nanotubes obtaining," In 4th European Congress of Chemical Engineering, 2004, vol. 9, pp. 161-162.

[6] E. G. Rakov, "Pyrolytic synthesis of carbon nanotubes and nanofibers," Russian chemical journal, vol. 48, no. 5, pp. 12-20, 2004.

[7] E. M. Koltsova, et al., "Development of a new technology for producing carbon nanotubes and pure hydrogen by catalytic pyrolysis of hydrocarbon raw materials," Science Magazine Modern High Technologies, vol.7, pp.141146, 2010.

[8] B. Ismailov, S. Meldebekova, Kh. Ismailov, and Y. Nametova, "A method for solving equations of the turbulent atmospheric diffusion," International Conference on Applied Analysis and Mathematical Modeling (ICAAM 2014), pp. 67-72, Sep. 2014, doi: 10.1063/1.4893806.

[9] B. Ismailov, Kh. Ismailov, A. Urmatova, and T. Koyshieva, "Mathematical Modelling, Dynamic and MassTransfer Calculation of Gas-Drop Mixture in the Mass-Transfer Apparatus Multistage Channels," Applied Mathematical Sciences, vol. 8, no.92, pp.4561-4570, 2013, doi: 10.12988/ams.2014.46471.

[10] B. Ismailov, A. Urmatova, and Kh. Ismailov, "Mathematical modeling and calculation of dynamic characteristics of gas in multistage channels," Applied Mathematical Sciences, vol. 7, no.132, pp. 6571-6582, 2013, doi: 10.12988/ams.2013.310561.

[11] B. Ismailov, A.Urmatova, and Kh.Ismailov, "Modeling and calculation of the trajectory of droplets in a multistage channel mass-transfer apparatus," In AIP International Conference on Applied Analysis and Mathematical Modeling (ICAAM-2014), 2014, pp.63-66, doi: 10.1063/1.4893805.

[12] A. Ashyralyev, A. S. Erdogan, and N. Arslan, "On the modified Crank-Nicholson difference schemes for parabolic equations with non-smooth data arising in biomechanics," International Journal for Numerical Methods in Biomedical Engineering, vol. 26, no. 5, 501-510, 2010, doi: 10.1002/cnm.1190.

[13] A. Ashyralyev, A. S. Erdogan and O. Demirdag, "On the determination of the right-hand side in a parabolic equation," Applied Numerical Mathematics, vol. 62, no. 11, pp. 1672-1683, Nov. 2012, doi: 10.1016/j.apnum.2012.05.008.

[14] A. Ashyralyev, and A. S. Erdogan, "Finite Difference Method for the Estimation of a Heat Source Dependent on Time Variable," Malaysian Journal of Mathematical Sciences, vol. 6, pp. 139-150, 2012.

[15] M. Ashyralyyeva, and A. Ashyralyyev, "Stability of implicit difference scheme for solving the identification problem of a parabolic equation,"In AIP Conference Proceedings, 2015, vol. 1676, no. 1, doi: 10.1063/1.4930450.

[16] A. Ashyralyev, A. S. Erdogan, "Well-posedness of the problem of right hand side identification for a parabolic equation," vol. 66, no.2, pp. 147-158, 2014, doi: 10.1007/s11253-014-0920-0.

[17] A. Ashyralyev, and A. Sarsenbi., "Well-posedness of a parabolic equation with nonlocal boundary condition," Boundary Value Problems, vol. 38, pp. 1-11, Dec. 2015, doi: 10.1186/s13661-015-0297-5.

[18] A. Ashyralyev, and A. Sarsenbi, "Well-Posedness of a Parabolic Equation with Involution," Numerical Functional Analysis and Optimization, vol. 38, no. 10, pp. 1295-1304, Jan. 2017, doi: 10.1080/01630563.2017.1316997.

[19] A. Ashyralyev, A. Erdogan, and A. S andazaklioglu, "Numerical solution of a source identification problem: almost coercivity," Inverse and Ill-posed Problems, vol. 27, no. 4, pp. 457-468, Sep. 2019, doi: 10.1515/jiip-2017-0072.

[20] A. Ashyralyev, and D. Agirseven, "On source identification problem for a delay parabolic equation," Nonlinear Analysis: Modelling and Control, vol. 19, no. 3, pp. 335-349, Sep. 2014, doi: 10.15388/NA.2014.3.2.

[21] A. Brener, V. Golubev, A. Kazenova, A. Sadyrbaeva, and G. Kenzhalieva, "Novel Considerations on the Scaling of Rate Kernels in the Equations of Cluster Aggregation," Journal of Physics Conference Series, vol. 1334, pp. 1-7, Oct. 2019, doi: 10.1088/1742-6596/1334/1/012013.

[22] L. Tashimov, A. Brener, A. Muratov, and G. Berdalieva, "Modeling the flows of thick suspensions and precipitations in technological apparatuses," In AIP Conference Proceedings, 2018, vol. 1982, pp. 1-6, doi: 10.1063/1.5045450.

[23] M. G. S. Bernd, S. R. Bragança, N. Heck, and L. C. da Silva Filho, "Synthesis of carbon nanostructures by the pyrolysis of wood sawdust in a tubular reactor," Journal of Materials Research and Technology, vol.6, no.2, pp. 171-177, Apr. 2017, doi: 10.1016/j.jmrt.2016.11.003.

[24] Paul T. Williams, "Hydrogen and Carbon Nanotubes from Pyrolysis-Catalysis of Waste Plastics: A Review," Waste and Biomass Valorization, vol. 12, pp. 1-28, Oct. 2021, doi: 10.1007/s12649-020-01054-w.

[25] H. E. Figen, and S. Z. Baykara, "Hydrogen production by partial oxidation of methane over Co based, Ni and Ru monolithic catalysts," Int. J. Hydrogen Energy, vol. 40, no.24, pp.7439-7451, Jun. 2018, doi: 10.1016/j.ijhydene.2015.02.109. 
[26] J. Goscianska, R. Pietrzak, and J. Matos., "Catalytic performance of ordered mesoporous carbons modified with lanthanides in dry methane reforming," Catalysis Today, vol. 301, pp. 204-216, Mar. 2018, doi: 10.1016/j.cattod.2017.05.014.

[27] V. A. Kirillov, A. B. Shigarov, N. A. Kuzin, V. V. Kireenkov, A. S. Braiko, and N. V. Burtsev, "Ni/MgO catalysts on structured metal supports for the air conversion of lower alkanes to synthesis gas," Kataliz v promyshlennosti, vol. 19, no. 5, pp. 351-363, Sep. 2019, doi: 10.18412/1816-0387-2019-5-351-363.

\section{BIOGRAPHIES OF AUTHORS}
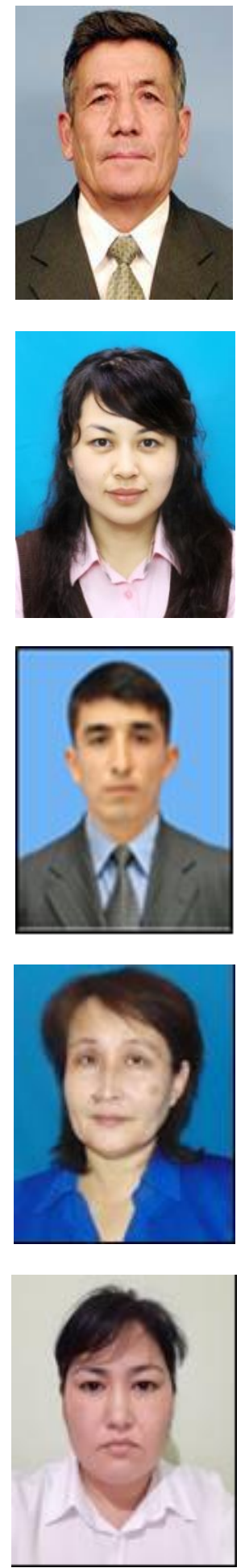

Bakhtiyar Rashidovich Ismailov is Doctor of Technical Sciences (2006), Professor of the Ministry of Education and Science of the Republic of Kazakhstan (2010). Currently, he works as a full professor at the Department of Information Systems and Modeling of the M. Auezov South Kazakhstan University. Scientific interests are represented in the following areas: mathematical and numerical modeling of phase interaction in contact devices of chemical technology devices; modeling and calculation of gas distribution in case of man-made emergencies; alternative energy.

Zhanat Rysbayevna Umarova received the PhD degree in Iformatics, Computer Engineering and Control from Ministry of Education and Science, in 2013. Currently she works as an associated professor of Information Systems and Modeling department at M. Auezov South Kazakhstan University. Research interests include mathematical modeling, computer simulation, information security and data protection in information systems, renewable recourses.

Khairulla Bakhtiyarovich Ismailov is candidate of Technical Sciences, Associate Professor of the Department of Information Systems and Modeling of M. Auezov South Kazakhstan University. In 2006 he defended his Ph.D. thesis on "Hydrodynamics and Mass Transfer in an Apparatus with Regular Vibrating Spiral Nozzles". Research interests: mathematical and computer modeling of chemical technology processes.

Aibarsha Abilkasymovna Dosmakanbetova is candidate of technical sciences (2011). Currently, she works as an associate professor of the department "Technological machines and equipment" of the South Kazakhstan University named after M.Auezov. Scientific interests are represented in the following areas: mathematical models of thermodynamic and heat and mass transfer processes; analysis of the problems of engineering design and methodology for calculating devices for producing ultrafine powder materials; kinetics of nucleation and aggregation of solid phase clusters during desublimation.

Saule Korgambaevna Meldebekova, Master of Mathematics (2005). Currently holds the position of senior lecturer of the Department of Computing and Software, South Kazakhstan University named after M.Auezov. Scientific interests are represented in the following areas: mathematical and numerical modeling of chemical technological processes; development of software for the implementation of mathematical models of the interaction of flows in technological devices. 